\title{
Análise do desempenho de um eletrocardiógrafo interpretativo para utilização em um sistema computacional de auxílio diagnóstico em síndromes coronarianas agudas
}

\author{
Manoel Gadêlha de Freitas Junior*, Denys Emilio Campion Nicolosi, \\ Francisco Faustino de Albuquerque Carneiro de França
}

Resumo O eletrocardiograma (ECG) registra a atividade elétrica cardíaca de forma não invasiva e permite obter uma avaliação rápida do ritmo cardíaco, muito útil na definição da conduta terapêutica imediata em pacientes com suspeita de síndrome coronariana aguda (SCA), que é uma entidade clínica que engloba a angina instável e os infartos agudos do miocárdio com e sem supra-desnível do segmento ST. Atualmente, eletrocardiógrafos de diversos fabricantes são capazes de fornecer diagnósticos automáticos dos ECGs, mas, apesar disso, existem poucos estudos publicados acerca do desempenho desses equipamentos. O objetivo deste trabalho é avaliar o desempenho de um eletrocardiógrafo interpretativo para determinar sua adequabilidade para uso em um sistema computacional que utiliza informações da história clínica obtida pelo médico generalista e do laudo eletrocardiográfico emitido pelo equipamento avaliado, permitindo auxiliar no diagnóstico de síndromes coronarianas agudas, quando o paciente não é atendido por cardiologistas. Foi utilizado um eletrocardiógrafo interpretativo Nihon Kohden Cardiofax 9620 que foi testado com um simulador de sinais eletrocardiográficos Metron PS 420 e, ainda, teve seu desempenho comparado com um sistema de telemedicina disponível em uma região semi-rural brasileira. Os testes resultaram em 93,8\% de sensibilidade e $83,3 \%$ de especificidade, que foram considerados satisfatórias em situações clínicas compatíveis com isquemia miocárdica aguda, validando a sua utilização em um sistema de inteligência artificial para auxílio ao diagnóstico em SCA.

Palavras-chave Eletrocardiograma (ECG), Eletrocardiógrafo interpretativo, Síndrome Coronariana Aguda (SCA).

\section{Performance analysis of an interpretive electrocardiograph for use in a computer-aided diagnostic system for acute coronary syndromes}

\footnotetext{
Abstract The electrocardiogram (ECG) is the recording of the heart's electrical activity from the surface of the body and has been worldwide used in routine medical practice since the last century. This is a first-rate tool for a rapid interpretation of cardiac rhythm and for assessment and immediate therapeutic management in patients with suspected acute coronary syndrome, which is a clinical entity that includes unstable angina and acute myocardial infarction with and without ST-segment elevation. Currently, electrocardiographs from various manufacturers are capable of providing automatic diagnosis of ECGs, but there are few published studies about the performance of these equipments. The objective of this paper is to evaluate the performance of an interpretive electrocardiograph in order to determine their suitability for use in a computer system that uses the medical history information obtained by the general practitioner and the electrocardiographic report emitted by the equipment evaluated to aid the diagnosis of acute coronary syndromes, when the patient is not treated by cardiologists. We used an interpretive electrocardigraph Nihon Kohden Cardiofax 9620. The electrocardiograph simulator Metron PS 420 was the choice for the simulated tests, and also we compared the performance of the Cardiofax with a telemedicine system available in a semi-rural area in Brazil. The tests resulted in $93.8 \%$ sensitivity and $83.3 \%$ specificity that were considered satisfactory in clinical situations compatible with acute myocardial ischemia, validating their use in an artificial intelligence system for the diagnosis of ACS.
}

Keywords Electrocardiogram (ECG), Interpretive electrocardiograph, Acute Coronary Syndrome (ACS).

*e-mail: manoelgadelha@hotmail.com

Recebido: 08/05/2011 / Aceito: 09/02/2012 


\section{Extended Abstract}

\section{Introduction}

The electrocardiogram (ECG) is the recording of the heart's electrical activity from the surface of the body and has been used worldwide in medical routine since the last century. This is a first-rate tool for a rapid interpretation of cardiac rhythm and for assessment and immediate therapeutic management in patients with suspected acute coronary syndrome, which is a clinical entity that includes unstable angina and acute myocardial infarction with and without ST-segment elevation. Currently, electrocardiographs from various manufacturers are capable of providing automatic diagnosis of ECGs, but there are few published studies about their performance. The objective of this paper is to evaluate the performance of an interpretive electrocardiograph in order to determine their suitability for use in a computer system to aid the diagnosis of acute coronary syndromes, when the patient is not treated by cardiologists.

\section{Material and Methods}

The interpretive electrocardiograph under test is the Cardiofax Nihon Kohden Model 9620, using the interpretation software ECAPS 12C, and was tested with an equipment capable of simulating various electrocardiographic signals, the Metron model PS-420 Patient Simulator. Tests were also made with patients in medical units of the brazilian semi-rural areas to assess whether the sample size of in simulated tests had statistical validity, comparing the proportions of correct interpretive electrocardiograph with a system of telemedicine available in the medical facility. The automatic interpretations were submitted for analysis to an experienced electrocardiograph (third author), considered the "gold standard" who did not know what was the result of the interpretation made by the equipment and the selection of the ECG signal simulated by Metron. To validate the interpretation "gold standard", the first author analyzed the results of the electrocardiograph automatic interpretations, based on Cardiofax User's Guide and watching the brazilian guidelines, besides counting with the aid of textbooks in the area and his own experience in Cardiology. The validation criteria of the cases were the agreement between the ECG interpretation of the "gold standard" and the first author. Tests were accomplished using the ECG simulator Metron, totaling 33 different waveforms, whose respective electric signals were applied to the Cardiofax involving multiple values (in $\mathrm{mV}$ ) of ST segment slopes (above and below), ventricular tachycardial supraventricular tachycardia, ventricular premature complexes, atrial fibrillation/ventricular, atrial flutter, missed beat, atrial tachycardia, nodal rhythm, bundle branch block left/right and tachycardia and sinus bradycardia. Besides these, tests were performed with 419 patients who underwent examination with the interpretive electrocardiograph in various clinical situations, outpatient and emergency.

\section{Results}

The statistical parameters obtained with the electrocardiograph interpretation are summarized in Tables 2-6, highlighting the sensitivity of $93.8 \%(95 \%$ CI 81.9 - 105.6) and specificity of $83.3 \%$ (95\% CI 66.1 - 100.6) for electrocardiographic findings consistent with ACS. In order to calculate the minimum number of samples required for validation, the performance of interpretive electrocardiograph was compared with that of a system of telemedicine available in a medical facility in a brazilian semi-rural area, whereas, in theory, this would be the best electrocardiographic interpretive capacity of a general physician in the emergency room. A total of 26 ECGs interpreted via telemedicine randomly chosen were analyzed and the results are shown in Tables 7 and 8. The difference between the rates of correct interpretation of the electrocardiogram and the telemedicine available was $35.65 \%(\alpha=0.05, \beta=0.9)$ in favor of Cardiofax. This is far superior the difference considered for the purposes of this study as statistically significant (15\%) for comparison of both diagnostic methods in order to make the calculation of the number of samples.

\section{Discussion}

Although only thirty three (33) tests were performed with the interpretative ECG, the results can be considered valid since that number was higher than the necessary to achieve statistical significance (25), considering the system of telemedicine available, used for comparison. Nevertheless, one cannot infer that an interpretive electrocardiograph always presents a better performance than a system of telemedicine. It is possible that, in this case, we have a comparison between an interpretive electrocardiograph of acceptable quality and a low quality telemedicine. Table 4 shows that all the statistics are within the confidence interval 95\% and the kappa statistics (0.77) can be considered satisfactory.

\section{Conclusion}

This paper demonstrates that the equipment under test has a rated performance that makes it suitable as an element of a computer-aided diagnostic system in acute coronary syndromes to be used in emergency situations where there are deficiencies in the interpretation of electrocardiograms. Moreover, the American Heart Association (AHA) and American College of Cardiology (ACC) consider reasonable the use of electrocardiograms which have validated algorithms to interpret computer generated for the pre-hospital diagnosis of acute coronary syndromes, with the level of evidence B, class IIa, (procedures categorized as "it is reasonable", "may be useful/effective/beneficial", "is probably recommended or indicated"). Additionally, it was identified a qualitative deficiency in a private telemedicine service, suggesting that this kind of medical service should be subjected to certification procedures and evaluated by a public audit mechanism. 


\section{Introdução}

As síndromes coronarianas agudas (SCAs) são entidades mórbidas que decorrem de processos isquêmicos no miocárdio, os quais, por sua vez, se devem a distúrbios do fluxo sanguíneo coronariano. Estas síndromes são responsáveis pela maior causa de mortalidade no mundo e a segunda no Brasil (Instituto Nacional de Cardiologia, 2008). A elevada taxa de mortalidade global, possivelmente, apresenta um componente que decorre de falhas diagnósticas, principalmente se for considerado que tipicamente o paciente com um quadro isquêmico cardíaco agudo é atendido em serviços de pronto-socorro (PS) por clínicos gerais, ou seja, em unidades de emergência não especializadas em Cardiologia.

Como o eletrocardiograma (ECG) é um meio prático, confiável e de baixo custo para obter uma interpretação rápida do ritmo cardíaco, surgiram nos últimos anos diversos sistemas de telemedicina capazes de interpretar remotamente esse exame, embora não possuam a capacidade de sugerir o diagnóstico da condição patológica que acomete o paciente; esse diagnóstico seria feito pelo médico in loco, considerando a história clínica e o resultado da interpretação remota do ECG. Apesar desta limitação, a telemedicina pode disponibilizar para situações emergenciais, valiosas informações quanto à avaliação da situação cardiovascular do paciente, desde que os eletrocardiogramas recebidos sejam interpretados corretamente (exigindo que seja feita por um cardiologista experiente) e que o seu resultado seja remetido em tempo hábil, pois em síndromes coronarianas agudas existe a máxima: "tempo é músculo [cardíaco]". Assim sendo, sistemas de telemedicina com deficiências qualitativas podem apresentar erros interpretativos e atrasos no envio do laudo eletrocardiográfico, com graves conseqüências para os pacientes com isquemia miocárdica aguda, pois uma parcela significativa deles morre na primeira hora decorrida desde o início dos sintomas (Oliveira e Spiandorello, 2001). A literatura mostra a dificuldade em identificar rapidamente tais pacientes, relatando que dos cerca de 8 milhões de casos anuais de dor torácica relatados em serviços de emergência, $50 \%$ foram diagnosticados como SCA e os outros $50 \%$ tiveram alta, sendo que, destes últimos, entre 2 e $5 \%$ correspondem às chamadas altas inadvertidas por falhas diagnósticas (Selker et al., 1993). Entretanto, enquanto muitos pacientes com isquemia miocárdica aguda são corretamente internados em um hospital, os que são liberados (altas inadvertidas) apresentam um risco de morte - no domicílio ou no retorno à unidade hospitalar - em torno de $25 \%$, que é o dobro do índice dos que foram internados (Pope et al., 2000).
Por outro lado, há que se mencionar que existem situações onde os sistemas de telemedicina não poderiam ser implantados, como em localidades com deficiências de recursos financeiros ou de infra-estrutura em sistemas de telecomunicação e/ou de energia elétrica. Adicionalmente, os médicos que lidam com pacientes com quadro clínico de SCA a bordo de navios, de plataformas marítimas de petróleo, de aeronaves comerciais e de resgate aeromédico, além das ambulâncias do SAMU (Serviço de Atendimento Móvel de Urgência) se beneficiariam do fato possuir um eletrocardiógrafo interpretativo capaz de interpretar corretamente e apenas alguns segundos um ECG compatível com isquemia miocárdica aguda. Como em síndromes coronarianas agudas é importante que se faça o seu diagnóstico - e consequente tratamento - no menor tempo possível, essa disponibilidade imediata do laudo eletrocardiográfico pode ser considerada como uma vantagem sobre a telemedicina cardiológica, em que o tempo de envio da interpretação do ECG é, geralmente, significativamente maior.

Por sua vez, os eletrocardiógrafos interpretativos possuem capacidade computacional de análise do ECG desenvolvida a partir de métodos estatísticos, inteligência artificial ou de regras pré-estabelecidas (Kors e van Bemmel, 1990). Os resultados interpretativos automáticos que dependem de medidas precisas das amplitudes e das durações do ECG, como, por exemplo, a detecção da hipertrofia ventricular esquerda, podem se aproximar bastante das interpretações feitas por cardiologistas experientes, em termos de sensibilidade, especificidade e reprodutibilidade (Jakobsson et al., 1989; Willems et al., 1991). Esta característica, por si só, aumenta a consistência do reconhecimento do infarto agudo do miocárdio, muito embora algoritmos para quantificação dessa condição clínica sejam fortemente dependentes de análise computacional para sua efetividade (Kligfield, 2005). Entretanto, interpretações que dependem da configuração da forma de onda, como alterações de repolarização, relações entre formas de onda e irregularidades na onda P, não foram consideradas muito precisas por alguns autores (Taha et al., 2000; Thomson et al., 1989) de tal forma que esforços foram feitos pelos fabricantes no sentido de melhorar o desempenho dos eletrocardiógrafos interpretativos nessas situações (Rautaharju, 1993; Taha et al., 2000; Wooley et al., 1992) com relativo sucesso (Farrell et al., 2003; Reddy et al., 1998; Spodick e Bishop, 1997; Taha et al., 2000).

O objetivo deste trabalho é avaliar o desempenho de um eletrocardiógrafo interpretativo para determinar sua adequabilidade para uso em um sistema computacional que utiliza informações da história clínica obtida pelo 
médico generalista e do laudo eletrocardiográfico emitido pelo equipamento avaliado, permitindo auxiliar no diagnóstico de síndromes coronarianas agudas, quando o paciente não é atendido por cardiologistas, estudando o seu desempenho na identificação de registros de ECG que possam ser compatíveis com quadros de síndromes coronarianas agudas, além de outros traçados relacionados com as falhas em diagnosticar essas síndromes. Uma importante fonte de falha diagnóstica nesses casos, detectada em estudo realizado nos Estados Unidos da América, correspondeu à inabilidade dos médicos do pronto-socorro em detectar elevações de 1 a $2 \mathrm{~mm}$ no segmento ST em ECGs de pacientes com infarto agudo do miocárdio (IAM), o que foi notado em $11 \%$ dos casos (Pope et al., 2000). Isso não ocorre com um eletrocardiógrafo interpretativo, pois a mensuração que ele faz dessas elevações do segmento ST é a partir de medidas reais de diferença da tensão elétrica entre a linha que precede o complexo QRS e a que o sucede, não dependendo das condições visuais (luz ambiental, acuidade da visão próxima ou presbiopia) que influenciam a análise do traçado do ECG feita por médico. Outros achados eletrocardiográficos, relacionadas com altas indevidas, em síndromes coronarianas agudas, são: 1) alterações inespecíficas de segmento ST ou anormalidades de onda $\mathrm{T} ; 2$ ) anormalidades secundárias de segmento ST (hipertrofia ventricular esquerda, bloqueio de ramo esquerdo, variante de repolarização precoce ou pericardite);3) cicatriz miocárdica prévia; e 4) eletrocardiograma com ritmo sinusal normal (Pope et al., 2000). Para todas essas situações, espera-se um desempenho que seja considerado aceitável para o eletrocardiógrafo interpretativo que esteja sendo utilizado por clínicos gerais no diagnóstico de síndromes coronarianas agudas. Por outro lado, como existem síndromes coronarianas agudas com eletrocardiograma normal, fica evidente que fazer o diagnóstico correto de um ritmo sinusal normal faz parte das características desejáveis para o equipamento, havendo relato, na literatura, de valores significativamente elevados de sensibilidade, especificidade, valor preditivo positivo e negativo para as interpretações automáticas de ritmo sinusal com esse tipo de eletrocardiógrafo (Poon et al., 2005).

A avaliação eletrocardiógrafo interpretativo sob teste também objetiva verificar sua adequação a um sistema computacional para auxílio ao diagnóstico de síndromes agudas coronarianas, em desenvolvimento, para ser utilizado por médicos não cardiologistas, atuando em locais com poucos recursos, especialmente aqueles sem capacidade de dosar marcadores de necrose miocárdica e sem acesso a sistemas de telemedicina de boa qualidade. Este sistema extrapola o simples fornecimento de um laudo eletrocardiográfico, ao agregar a interpretação do ECG com dados da história clínica do paciente, fornecendo, assim, uma sugestão diagnóstica para o seu quadro clínico, auxiliando in loco o médico generalista na tomada de decisão e subsequente conduta terapêutica. Por sua aplicabilidade em serviços médicos de emergência sem especialistas em Cardiologia, fixos ou móveis, terrestres ou não, o sistema em desenvolvimento poderá contribuir para reduzir a taxa de mortalidade cardiovascular global, especialmente nas regiões mais carentes do Brasil e do Mundo, através da minimização do índice de falhas diagnósticas dos casos de SCA atendidos por esses serviços.

\section{Materiais e Métodos}

Os materiais e os métodos utilizados neste trabalho foram analisados pelo Comitê de Ética em Pesquisa (CEP) do Instituto Dante Pazzanese de Cardiologia, consistindo de registros de sinais eletrocardiográficos de pacientes ou simulados. O eletrocardiógrafo interpretativo avaliado foi da marca Nihon Kohden modelo Cardiofax 9620, cuja acurácia atende aos padrões internacionais IEC6061-2-51. Trata-se de registrador em tempo real com 3 canais do sinal de ECG, o qual apresenta simultaneamente as formas de onda desse sinal em 12 derivações, com capacidade de armazenar até 8 registros em uma memória interna. É um equipamento compacto, cujas maiores dimensões externas (largura $\times$ comprimento) correspondem ao tamanho de uma folha de papel A4, e que imprime tanto o ECG quanto sua interpretação em rolo de papel termo-sensível de $63 \mathrm{~mm}$ de largura, facilmente disponível em lojas de material médico-hospitalar. Possui, ainda, uma tela de cristal liquido que possibilita um preview a cada $2,8 \mathrm{~s}$ do eletrocardiograma, podendo, assim, funcionar, também, como monitor de 12 derivações em tempo real, com a característica adicional de possuir comandos operacionais extremamente simples, permitindo o treinamento rápido de quem vai fazer uso do equipamento. Outra característica bastante interessante é a capacidade do equipamento de operar com uma bateria interna, capacitando-o a efetuar até 60 exames em situações de indisponibilidade de energia elétrica ou de deficiências de aterramento que aumentem significativamente o ruído de $60 \mathrm{~Hz}$.

O programa de interpretação do ECG usado no citado equipamento é próprio da Nihon Kohden, tendo recebido a denominação de ECAPS 12C. O fabricante adverte que o mesmo só é aplicável para pacientes com idades acima de três anos e que a interpretação resultante do programa deve ser submetida ao julgamento de um médico qualificado, para a sua validação. Essa validação tornar-se-ia 
necessária considerando que um programa de análise computacional baseia-se em uma coleção de critérios de avaliação de ECG estabelecido por médicos, de tal forma que, eventualmente, pode não ser possível para esse programa julgar corretamente determinado registro eletrocardiográfico em uma hipotética situação em que um médico habilitado o faria de maneira consistente. As limitações do programa ECAPS 12C incluem (Nihon Kohden, 2009):

- não compensa a influência de uso de determinados medicamentos; a influência de digitálicos pode ser notada no registro, por exemplo, pelo diagnóstico de fibrilação atrial;

- não considera distúrbios hidroeletrolíticos, recomendando, no julgamento médico do ECG, fazer a leitura do intervalo QTc, onda T e onda $U$, juntamente com outros elementos relevantes;

- não classifica complexos prematuros como trigeminados, "short-run", etc.

- não é capaz de identificar batimentos de escape e para-arritmias, sendo esses considerados como "ritmos indeterminados";

- não diagnostica a Síndrome de Lown-GaningLevine; esses casos devem ser considerados a partir do diagnóstico de "intervalo PR curto";

- não identifica marcapassos do tipo "wandering" (atrial migratório).

Considerando a utilização que se pretende fazer do equipamento, essas limitações não foram consideradas como significativas, pois é requerida primariamente a correta identificação de desníveis de segmento ST e de outras anomalias eletrocardiográficas passíveis de serem encontradas em síndromes coronarianas agudas, as quais não se inserem, em princípio, nessas restrições do desempenho interpretativo.

O Cardiofax foi testado nos Laboratórios de Bioengenharia do Instituto Dante Pazzanese de Cardiologia, utilizando um equipamento capaz de simular diversos sinais eletrocardiográficos, incluindo aqueles apresentados nas síndromes coronarianas agudas, o Metron modelo PS-420 Patient Simulator. Também foram realizados testes com pacientes em unidades médicas semi-rurais (pequenas cidades) do Nordeste, estes para avaliar se o tamanho da amostra da simulação com o Metron possuía validade estatística, comparando as proporções de acerto do eletrocardiógrafo interpretativo com as de um sistema de telemedicina disponível na unidade. As interpretações automáticas foram submetidas à apreciação de um eletrocardiografista experiente, responsável pelo Serviço de Telemedicina do Instituto Dante Pazzanese de Cardiologia, considerado como "gold standard", resultando em um teste cego, por não saber qual era o resultado da interpretação feita pelo equipamento nem a seleção do sinal elétrico simulado pelo Metron. A título de validação dos padrões de interpretação, além do julgamento do eletrocardiografista experiente ("gold standard"), o primeiro autor analisou os resultados das interpretações automáticas do eletrocardiógrafo, baseando-se nas orientações contidas no User's Guide do Cardiofax 9620 e respeitando as diretrizes brasileiras vigentes para a interpretação de eletrocardiogramas (Sociedade Brasileira de Cardiologia, 2009), além de contar com as orientações obtidas de livros texto na área (Deccache, 2006; Frieden e Ira, 1974; Hampton e Adlam, 2009) e com a própria experiência médica em Cardiologia. A mesma sistemática foi adotada para a análise dos laudos eletrocardiográficos emitidos pela telemedicina. Foram aceitos como válidos os casos em que houve concordância entre as opiniões do "gold standard" e do primeiro autor quanto à interpretação do ECG, tanto nas interpretações automáticas quanto naquelas do sistema de telemedicina.

Com relação aos testes feitos em pacientes, o eletrocardiógrafo interpretativo foi utilizado para obter ECGs de pessoas que se apresentaram em ambulatórios ou em serviços de emergência urbanos (capital) e semi-rurais (pequenas cidades) em um estado da região Nordeste do Brasil. Esses exames, solicitados por ordem médica, eram realizados de forma duplicada, um contendo a identificação completa do paciente, que era entregue ao médico e outro, contendo apenas as iniciais, o sexo e a idade do mesmo, além de dados essenciais quanto ao quadro clínico, que era retido para a pesquisa.

Os diagnósticos eletrocardiográficos gerados pelo Cardiofax em pacientes foram classificados em cinco categorias, adaptando uma padronização disponível na literatura (Snyder et al., 2003) considerando as peculiaridades do eletrocardiógrafo em uso, cujos laudos são impressos em inglês, de modo que alguns diagnósticos sofreram alteração na sua tradução, visando um melhor entendimento na linguagem médica brasileira. Além disso, para facilitar o trabalho estatístico, diagnósticos com determinadas similaridades foram agrupados em um único bloco (exemplo: extra-sístoles ventriculares e supra-ventriculares, consideradas apenas como "extra-sístoles").

O programa ECAPS 12C do Cardiofax utiliza uma modificação do Código de Minnesota (Nihon Kohden, 2009) adotado pela Organização Mundial de Saúde para a classificação de formas de onda eletrocardiográficas, de modo que as interpretações do programa podem diferir daquelas estabelecidas nesse código. Além disso, as interpretações automáticas desse equipamento são 
frequentemente apresentadas na forma de uma lista - às vezes extensa - de diagnósticos eletrocardiográficos, de modo que, em certos casos, aqueles precedidos das palavras "possível", "provável" foram desconsiderados, atendo-se no máximo a dois diagnósticos relevantes (o próprio programa ECAPS $12 \mathrm{C}$ os lista em ordem decrescente de importância clínica) e à conclusão final se o ECG era "normal", "anormal" ou "limítrofe". $\mathrm{Na}$ operação do eletrocardiógrafo interpretativo, a presença de "artefatos" (ruído de $60 \mathrm{~Hz}$, deslocamento da linha de base, sinais eletromiográficos, etc.) pode conduzir a diagnósticos errôneos, de modo que técnicas adequadas de manuseio devem ser obedecidas pelos operadores do equipamento, visando minimizar esses efeitos deletérios.

Por outro lado, as Diretrizes da AHA/ACC para o manejo de pacientes com infarto agudo do miocárdio (Anderson et al., 2007) estabelecem, em algoritmo para o manejo de pacientes com sintomatologia compatível com doença miocárdica aguda de caráter isquêmico, que uma das primeiras condutas médicas seja a avaliação do ECG de 12 derivações inicial, o qual pode se apresentar em uma das seguintes situações:

- elevação do segmento ST;

- bloqueio de ramo novo ou presumivelmente novo, usualmente comprometendo o ramo esquerdo do Feixe de His;

- depressão de ST, inversão de onda T (forte suspeita de isquemia miocárdica);

- normal ou não diagnóstico.

De modo a abranger todos esses diagnósticos eletrocardiográficos, foram feitos testes com o equipamento simulador de sinais eletrocardiográficos Metron, totalizando 33 formas de onda eletrocardiográficas geradas por esse equipamento, cujos respectivos sinais elétricos foram aplicadas aos cabos do Cardiofax, englobando diversos valores (em $\mathrm{mV}$ ) de desníveis de segmento ST (supra e infra), taquicardia ventricular/supraventricular, complexos ventriculares prematuros, fibrilação atrial/ventricular, flutter atrial, "missed beat", taquicardia atrial, ritmo nodal, bloqueios de ramo direito/esquerdo e taquicardia e bradicardia sinusal. Além desses, foram realizados testes com 419 pacientes que foram submetidos ao exame com o eletrocardiógrafo interpretativo, em diversas situações clínicas, ambulatoriais e emergenciais. As figuras de mérito estatístico consideradas foram a sensibilidade, a especificidade, o valor preditivo positivo, o valor preditivo negativo, a acurácia, o odds pré-teste, as relação de verossimilhanças (likelihood ratios) pré-teste positivo e pré-teste negativo, além da estatística kappa. Todas elas foram obtidas a partir de uma planilha Excel de cálculos estatísticos disponível na internet (Braile e Godoy, 1999), simplesmente lançando na mesma os valores da matriz de confusão dos testes, composta pelos falsos positivos e negativos e verdadeiros positivos e negativos, resultantes das comparações dos resultados das interpretações automáticas com aquelas do "gold standard" validadas pelo primeiro autor.

Para verificar se a quantidade de amostras obtidas (33) para este teste seria estatisticamente significante, existe a necessidade de verificar se ela atende ao critério do tamanho mínimo de amostras, discutido mais adiante neste trabalho. Para tanto, foi feita a comparação do desempenho do eletrocardiógrafo interpretativo com aquele de um sistema de telemedicina disponível em uma unidade médica semi-rural do Brasil, considerando que, em tese, essa seria a melhor capacidade interpretativa eletrocardiográfica do médico clínico geral em um pronto socorro não especializado, levando em conta que geralmente eles possuem pouca habilidade nesse particular e que uma interpretação via telemedicina seria o ápice da capacidade de diagnóstico eletrocardiográfico do serviço médico semi-rural brasileiro. Um total de 26 eletrocardiogramas interpretados através do sistema de telemedicina disponível foi escolhido aleatoriamente e submetidos ao eletrocardiografista "gold standard", número que, conforme se verá mais adiante, atende ao critério do tamanho mínimo de amostras. O interesse doravante limita-se à determinação das taxas de acertos dos testes diagnósticos eletrocardiográficos avaliados, para poder efetuar o cálculo do tamanho mínimo de amostras, e, assim, caso satisfeito esse número, aceitar os resultados dos testes simulados com o Metron.

\section{Critérios de inclusão}

Foram incluídos registros eletrocardiográficos de pacientes:

- de ambos os sexos, com idade superior a 3 anos, sem limite superior de idade;

- assintomáticos ou sintomáticos; e

- em situações ambulatoriais ou emergenciais.

\section{Critérios de exclusão}

Foram excluídos desta investigação os casos em que o eletrocardiograma:

- apresentasse evidências de ter havido a colocação incorreta de eletrodos;

- contivesse um nível de ruído capaz de comprometer a interpretação automática; e

- estivesse ilegível, na forma de onda registrada ou no(s) diagnóstico(s) resultante(s) da interpretação automática. 


\section{Cálculo do tamanho mínimo de amostras}

Este trabalho apresenta características compatíveis com um experimento clínico controlado que não submete o paciente a efeitos terapêuticos de medicamentos, mas ainda assim retém elementos delineativos desse tipo de estudo. Existe, portanto, a necessidade de se conhecer o tamanho mínimo das amostras estatisticamente significante, antes de realizar a casualização dos grupos, um submetido ao método diagnóstico A (eletrocardiógrafo interpretativo) e outro ao método diagnóstico B, que, no caso, seria a interpretação feita por um clínico geral atuando em uma unidade médica com poucos recursos diagnósticos. Na ausência de estatísticas nacionais a respeito do desempenho desses profissionais, a interpretação do ECG dada por um serviço de telemedicina disponível na região semi-rural foi assumida como sendo igual à do médico que estaria atendendo ao paciente, e esse serviço foi considerado como sendo o método B. Desta forma, há a necessidade que se estabeleça as seguintes definições, considerando-se eventos que, estatisticamente, tenham uma distribuição normal: a) qual é a proporção de acertos diagnósticos com o método $\mathrm{B}$; b) qual é a diferença mínima, entre a proporção de acertos com o novo método A e o método $\mathrm{B}$, considerado como de significado clínico para que se recomende o método A; c) definição da probabilidade de erro tipo I $(\alpha)$ e erro tipo II $(\beta)$. Desta forma, os parâmetros utilizados para o cálculo do tamanho mínimo da amostra foram: a) proporção dos grupos $(A=89,5 \%$ e $\mathrm{B}=53,85 \%)$, b) nível de significância de 5\%, c) poder do teste de $90 \%$, e d) teste de hipótese monocaudal, pois se trata de um teste cuja hipótese alternativa é uma desigualdade, ou seja, deseja-se testar se o valor observado é maior ou menor que o valor crítico correspondente à hipótese nula (Instituto Dante Pazzanese de Cardiologia, 2010).

Assim sendo, a determinação do tamanho mínimo de amostras, neste estudo, enquadra-se como "comparação de duas proporções", no caso, aquela dos acertos do método A quando comparados a dos acertos do método B. Na comparação de proporções de duas populações, o pesquisador observa as proporções em dois grupos distintos e as compara com o objetivo de saber se os grupos diferem ou não em relação à resposta de interesse. Para este tipo de análise é assumido que o número de observações seria o mesmo nas duas amostras. As proporções de acerto associadas aos métodos, acima mencionadas, foram obtidas através das observações feitas sobre o desempenho dos mesmos, detalhadas mais adiante. $\mathrm{O}$ resultado para o tamanho mínimo da amostra, com os parâmetros acima, pode ser obtido diretamente no site http://www.lee.dante.br (Instituto Dante Pazzanese de
Cardiologia, 2010), sendo, no presente caso, apenas 25 pacientes por grupo, para $\alpha=0,05$ e $\beta=0,9$.

\section{Resultados}

Na Tabela 7 nota-se um baixo índice global de acertos da interpretação dos eletrocardiogramas, quando emitida por determinado serviço de telemedicina contratado por uma unidade médica semi-rural, contrapondo-se com o percentual razoável de acertos dos laudos emitidos pelo eletrocardiógrafo interpretativo, índices esses obtidos de informações que serão apresentadas mais adiante.

Analisando-se os resultados da Tabela 1, percebe-se que, para as situações de eletrocardiograma com supra-desnível de ST, o eletrocardiógrafo interpretativo acerta sete em oito situações, ou seja, uma sensibilidade de $87,5 \%$. É notável o erro do "gold standard" quando o supra-desnível simulado pelo Metron é muito pequeno $(0,1 \mathrm{mV}$ ou $1 \mathrm{~mm})$, considerando o ECG como "normal". O eletrocardiógrafo interpretativo, por mensurar voltagem diretamente, ao rotular esse caso como "anormalidade inespecífica de ST e anormalidade da onda T", consegue, neste caso, superar o desempenho do cardiologista experiente devido às dificuldades de visualização de um desnível desta ordem de grandeza quando impresso em um papel.

Na Tabela 2 são apresentados os resultados obtidos com os sinais simulados com o Metron para as situações de infra-desnível de ST, com um índice de acertos diagnósticos (sensibilidade) de 100\%.

Além dessas situações compatíveis com isquemia miocárdica, outras condições clínicas foram simuladas, e em um total de 33 casos, 16 correspondiam a formas de onda encontradas em isquemia miocárdica aguda, com as demais simulando outras condições patológicas. Das condições compatíveis com isquemia houve apenas um falso positivo, enquanto nas outras condições (não-isquemia) foram constatados três falsos negativos.

De posse dessas informações, foi possível efetuar os cálculos estatísticos básicos para o teste do eletrocardiógrafo interpretativo, cujos resultados estão apresentados na Tabela 3 .

Observando o desempenho global em pacientes, (Tabelas 5 e 7) observa-se que há uma diferença significativa nas porcentagens globais de acerto do Cardiofax $(89,50 \%)$ e do serviço de telemedicina $(53,85 \%)$ utilizado para comparação. Isso confirma a adequabilidade do tamanho mínimo de amostras de 25 para cada grupo, previamente calculado neste trabalho. Esse número foi atendido tanto para o sistema de telemedicina observado (26 amostras) quanto para o eletrocardiógrafo interpretativo (419 amostras obtidas em pacientes e 33 amostras obtidas por simulação), o que confere validade estatística aos resultados obtidos 
Tabela 1. Desempenho do eletrocardiógrafo interpretativo na detecção de supra-desnível simulado de segmento ST (significância clínica definitiva).

Table 1. Performance of interpretive electrocardiograph to detect simulated ST elevation (definite clinical significance).

\begin{tabular}{|c|c|c|c|c|}
\hline \multirow{2}{*}{$\begin{array}{l}\text { Sinal eletrocardiográfico } \\
\text { simulado (supra-desnível } \\
\text { de ST em mV) }\end{array}$} & \multicolumn{2}{|c|}{ Resultado da análise do ECG } & \multirow{2}{*}{$\begin{array}{l}\text { Concordância } \\
\text { do autor com } \\
\text { o diagnóstico } \\
\text { do Gold } \\
\text { Standard e da } \\
\text { interpretação } \\
\text { automática }\end{array}$} & \multirow{2}{*}{$\begin{array}{l}\text { Resultado } \\
\text { final da } \\
\text { interpretação } \\
\text { automática }\end{array}$} \\
\hline & Interpretação automática & Gold Standard & & \\
\hline 0,8 & $\begin{array}{l}\text { Lesão ou infarto ântero-lateral e } \\
\text { inferior. ECG anormal }\end{array}$ & $\begin{array}{l}\text { Corrente de lesão } \\
\text { sub-epicárdica anterior } \\
\text { extensa e inferior. }\end{array}$ & Sim & Acerto \\
\hline 0,7 & $\begin{array}{l}\text { Lesão ou infarto ântero-lateral. } \\
\text { Possível lesão septal ou IAM, } \\
\text { lesão ou IAM inferior. ECG } \\
\text { anormal }\end{array}$ & $\begin{array}{l}\text { Corrente de lesão } \\
\text { sub-epicárdica anterior } \\
\text { extensa e inferior. }\end{array}$ & Sim & Acerto \\
\hline 0,6 & $\begin{array}{l}\text { Lesão ou infarto ântero-lateral. } \\
\text { Possível lesão septal ou IAM, } \\
\text { lesão ou IAM inferior. ECG } \\
\text { anormal }\end{array}$ & $\begin{array}{l}\text { Corrente de lesão } \\
\text { sub-epicárdica anterior } \\
\text { extensa e inferior. }\end{array}$ & Sim & Acerto \\
\hline 0,5 & $\begin{array}{l}\text { Lesão ou infarto ântero-Lateral. } \\
\text { Lesão ou infarto inferior. ECG } \\
\text { anormal }\end{array}$ & $\begin{array}{l}\text { Corrente de lesão } \\
\text { sub-epicárdica anterior } \\
\text { extensa e inferior. }\end{array}$ & Sim & Acerto \\
\hline 0,4 & $\begin{array}{l}\text { Lesão ou infarto ântero-Lateral. } \\
\text { Lesão ou infarto inferior. ECG } \\
\text { anormal }\end{array}$ & $\begin{array}{l}\text { Corrente de lesão } \\
\text { sub-epicárdica anterior } \\
\text { extensa e inferior. }\end{array}$ & Sim & Acerto \\
\hline 0,3 & $\begin{array}{l}\text { Possível lesão ântero-lateral ou } \\
\text { infarto agudo. Lesão inferior ou } \\
\text { infarto agudo. ECG anormal }\end{array}$ & $\begin{array}{l}\text { Corrente de lesão } \\
\text { sub-epicárdica anterior } \\
\text { extensa e inferior. }\end{array}$ & Sim & Acerto \\
\hline 0,2 & $\begin{array}{l}\text { Possível pericardite aguda. } \\
\text { ECG anormal }\end{array}$ & $\begin{array}{l}\text { Corrente de lesão } \\
\text { sub-epicárdica anterior } \\
\text { extensa e inferior. }\end{array}$ & Não & Erro \\
\hline 0,1 & $\begin{array}{l}\text { Anormalidade inespecífica de } \\
\text { ST e anormalidade da onda T. } \\
\text { ECG anormal }\end{array}$ & Normal. & Não & Acerto \\
\hline
\end{tabular}

quanto ao desempenho do Cardiofax, mostrado na Tabela 3, para as condições de trabalho em unidades médicas com deficiências na capacidade de diagnóstico eletrocardiográfico em síndromes coronarianas agudas.

É notável, dos resultados acima mostrados, o fato de que o índice global de acertos do eletrocardiógrafo interpretativo representou uma diferença, para melhor, de $35,65 \%$ ( $\alpha=5 \%, \beta=90 \%$ ), quando comparada com aquele do sistema de telemedicina disponível na unidade médica semi-rural observada.

\section{Discussão}

Evidentemente, dos resultados encontrados neste trabalho, não se pode inferir que um eletrocardiógrafo interpretativo apresentaria sempre um desempenho melhor do que um sistema de telemedicina; é possível que, neste caso, tenha existido a comparação entre um eletrocardiógrafo interpretativo de qualidade aceitável e uma telemedicina com sérias deficiências operacionais.
A diferença encontrada entre as taxas de acertos dos métodos, a favor do Cardiofax - quando comparada com o melhor método de interpretação eletrocardiográfica disponível para um paciente atendido por um clínico geral em um hospital semi-rural em uma região carente é muito superior à diferença considerada para os propósitos deste trabalho como estatisticamente significante para a comparação dos métodos diagnósticos, tornando aceitáveis os testes do eletrocardiógrafo interpretativo feitos com o simulador de sinais de ECG, pois, apesar do número aparentemente pequeno de testes simulados (33), este é superior ao número mínimo de amostras para se obter significância estatística (25). Mais ainda, é razoável pensar que o desempenho sofrível dos profissionais que interpretaram os eletrocardiogramas do serviço de telemedicina observado, seria o ápice do desempenho de um clínico geral, em uma unidade médica semi-rural, na interpretação de um ECG sem o auxílio de telemedicina, conferindo consistência à escolha do método comparativo e às conclusões deste trabalho. 
Tabela 2. Desempenho do eletrocardiógrafo interpretativo na detecção de infra-desnível simulado de segmento ST (significância clínica definitiva).

Table 2. Performance of interpretive electrocardiograph to detect simulated ST depression (definite clinical significance).

\begin{tabular}{|c|c|c|c|c|}
\hline \multirow{2}{*}{$\begin{array}{l}\text { Sinal eletrocardiográfico } \\
\text { simulado (infra-desnível } \\
\text { de ST em } \mathrm{mV} \text { ) }\end{array}$} & \multicolumn{2}{|c|}{ Resultado da análise do ECG } & \multirow{2}{*}{$\begin{array}{l}\text { Concordância } \\
\text { do autor com o } \\
\text { diagnóstico do } \\
\text { Gold Standard e } \\
\text { da interpretação } \\
\text { automática }\end{array}$} & \multirow{2}{*}{$\begin{array}{l}\text { Resultado } \\
\text { final da } \\
\text { interpretação } \\
\text { automática }\end{array}$} \\
\hline & Interpretação automática & Gold Standard & & \\
\hline 0,8 & $\begin{array}{l}\text { Infradesnível significativo } \\
\text { de ST, compatível com lesão } \\
\text { sub-endocárdica. ECG anormal }\end{array}$ & $\begin{array}{l}\text { Corrente de lesão } \\
\text { sub-endocárdica anterior } \\
\text { extensa e inferior }\end{array}$ & Sim & Acerto \\
\hline 0,7 & $\begin{array}{l}\text { Infradesnível significativo de } \\
\text { ST, compatível com lesão sub- } \\
\text { endocárdica. ECG anormal }\end{array}$ & $\begin{array}{l}\text { Corrente de lesão } \\
\text { sub-endocárdica anterior } \\
\text { extensa e inferior }\end{array}$ & Sim & Acerto \\
\hline 0,6 & $\begin{array}{l}\text { Infradesnível significativo de } \\
\text { ST, compatível com lesão sub- } \\
\text { endocárdica. ECG anormal }\end{array}$ & $\begin{array}{l}\text { Corrente de lesão } \\
\text { sub-endocárdica anterior } \\
\text { extensa e inferior }\end{array}$ & Sim & Acerto \\
\hline 0,5 & $\begin{array}{l}\text { Infradesnível significativo de } \\
\text { ST, compatível com lesão sub- } \\
\text { endocárdica. ECG anormal }\end{array}$ & $\begin{array}{l}\text { Corrente de lesão } \\
\text { sub-endocárdica anterior } \\
\text { extensa e inferior }\end{array}$ & Sim & Acerto \\
\hline 0,4 & $\begin{array}{l}\text { Infradesnível significativo de } \\
\text { ST, compatível com lesão sub- } \\
\text { endocárdica. ECG anormal }\end{array}$ & $\begin{array}{l}\text { Corrente de lesão } \\
\text { sub-endocárdica anterior } \\
\text { extensa e inferior }\end{array}$ & Sim & Acerto \\
\hline 0,3 & $\begin{array}{l}\text { Infradesnível significativo } \\
\text { de ST. Possível lesão } \\
\text { subendocárdica. ECG anormal }\end{array}$ & $\begin{array}{l}\text { Corrente de lesão } \\
\text { sub-endocárdica anterior } \\
\text { extensa e inferior }\end{array}$ & Sim & Acerto \\
\hline 0,2 & $\begin{array}{l}\text { Infradesnível significativo } \\
\text { de ST. Possível lesão } \\
\text { subendocárdica. ECG anormal }\end{array}$ & $\begin{array}{l}\text { Corrente de lesão } \\
\text { sub-endocárdica anterior } \\
\text { extensa e inferior }\end{array}$ & Sim & Acerto \\
\hline 0,1 & $\begin{array}{l}\text { Depressão moderada de ST. } \\
\text { ECG anormal }\end{array}$ & Normal & Não & Acerto \\
\hline
\end{tabular}

Tabela 3. Estatísticas do teste do eletrocardiógrafo interpretativo.

Table 3. Statistics of interpretive electrocardiograph test.

\begin{tabular}{|c|c|c|}
\hline Parâmetro & Resultado & Intervalo de confiança $95 \%$ \\
\hline Sensibilidade & $93,80 \%$ & $81,9-105,6$ \\
\hline Especificidade & $83,30 \%$ & $66,1-100,6$ \\
\hline Valor preditivo positivo & $83,30 \%$ & $66,1-100,6$ \\
\hline Valor preditivo negativo & $97,80 \%$ & $81,9-105,6$ \\
\hline Acurácia & $88,20 \%$ & $77,4-99,1$ \\
\hline Odds pré-teste & 0,889 & - \\
\hline Likelihood ratio pré-teste positivo & 5,6 & - \\
\hline Likelihood ratio pré-teste negativo & 0,1 & - \\
\hline Estatística kappa (grau de concordância corrigido) & 0,77 & $0,55-0,98$ \\
\hline
\end{tabular}

Por outro lado, analisando as informações constantes da Tabela 3, é possível constatar que todos os números encontrados para os parâmetros avaliados estão dentro do intervalo de confiança $95 \%$ e que o grau de concordância corrigido $(0,77)$ pode ser considerado bom. Em síntese, os cálculos estatísticos básicos, cujos resultados estão sumarizados nessa tabela mostram que o equipamento avaliado apresenta um desempenho que o torna recomendável como elemento constitutivo de um sistema de auxílio ao diagnóstico em síndromes coronarianas agudas que será utilizado em situações emergenciais nas quais existam deficiências na interpretação de eletrocardiogramas e sem dispor de sistemas de telemedicina.

Uma informação importante, obtida da Tabela 1, que simulou ECGs compatíveis com infarto agudo do miocárdio com supra-desnível de ST (de mortalidade elevada na primeira hora decorrida desde o início dos sintomas), é que todos os registros interpretativos 
do Cardiofax finalizaram com a indicação "ECG anormal", o que, mesmo no único caso de erro interpretativo encontrado ("possível pericardite aguda"), esse erro pode ser minimizado, pois no sistema de auxílio ao diagnóstico que fará uso do eletrocardiógrafo interpretativo a situação "ECG anormal" receberá uma pontuação diferente de zero. Além disso, o diagnóstico eletrocardiográfico "alterações inespecíficas de ST-T", associado ao supra-desnível mínimo de ST simulado no Metron, pode ser encontrado em casos de isquemia miocárdica aguda (Pope et al., 2000).

Tabela 4. Resultados dos diagnósticos eletrocardiográficos automáticos em pacientes, por classe de significância.

Table 4. Results of automated electrocardiograph diagnoses for patients, by class of significance.

\begin{tabular}{|c|c|c|c|c|c|c|}
\hline \multirow{2}{*}{ Classe } & \multirow{2}{*}{ Diagnóstico } & \multicolumn{3}{|c|}{ Quantidades } & \multicolumn{2}{|c|}{ Porcentagem (\%) } \\
\hline & & Acertos & Erros & Total & Acertos & Erros \\
\hline \multirow{3}{*}{ I } & Normal & & & & & \\
\hline & Ritmo sinusal & 385 & 0 & 385 & 100,00 & 0,00 \\
\hline & Total & 385 & 0 & 385 & 100,00 & 0,00 \\
\hline \multirow{11}{*}{ II } & Significância mínima & & & & & \\
\hline & Bradicardia sinusal & 4 & 2 & 6 & 66,67 & 33,33 \\
\hline & Taquicardia sinusal & 20 & 0 & 20 & 100,00 & 0,00 \\
\hline & Arritmia sinusal & 33 & 0 & 33 & 100,00 & 0,00 \\
\hline & Desvio do eixo elétrico com significância mínima & 4 & 0 & 4 & 100,00 & 0,00 \\
\hline & Bloqueio incompleto de ramo & 12 & 4 & 16 & 75,00 & 25,00 \\
\hline & Distúrbio de condução/ repolarização precoce & 19 & 4 & 23 & 82,16 & 17,39 \\
\hline & Artefato & 135 & 7 & 142 & 95,07 & 4,93 \\
\hline & Anormalidade inespecífica de ST-T & 49 & 9 & 58 & 88,48 & 15,52 \\
\hline & Atraso de condução inespecífico & 3 & 0 & 3 & 100,00 & 0,00 \\
\hline & Total & 279 & 26 & 305 & 91,48 & 8,52 \\
\hline \multirow{14}{*}{ III } & Significância clínica indeterminada & & & & & \\
\hline & Bloq. divisional/desvio anormal de eixo à esquerda & 29 & 4 & 33 & 87,88 & 12,12 \\
\hline & Aumento de dimensões atriais & 16 & 15 & 31 & 51,61 & 48,39 \\
\hline & Hipertrofia ventricular & 27 & 19 & 46 & 58,07 & 41,30 \\
\hline & Bloqueio completo de ramo direito & 9 & 3 & 12 & 75,00 & 25,00 \\
\hline & Bloqueio $\mathrm{AV}$ de $1^{\circ}$ grau & 11 & 1 & 12 & 91,67 & 8,33 \\
\hline & Extra-sístoles & 13 & 1 & 14 & 92,86 & 7,14 \\
\hline & Ritmo juncional & 2 & 3 & 5 & 40,00 & 60,00 \\
\hline & QRS de baixa voltagem & 3 & 0 & 3 & 100,00 & 0,00 \\
\hline & Elevação/depressão inespecífica/repol. precoce ST & 3 & 1 & 4 & 75,00 & 25,00 \\
\hline & Inversão de onda $\mathrm{T}$ & 16 & 3 & 19 & 84,21 & 15,79 \\
\hline & PR curto & 8 & 1 & 9 & 88,89 & 11,11 \\
\hline & Atraso de condução em VD (RSR em V1/V2) & 35 & 5 & 40 & 87,50 & 12,50 \\
\hline & Total & 172 & 56 & 228 & 75,44 & 24,56 \\
\hline \multirow{12}{*}{ IV } & Significância clínica definitiva & & & & & \\
\hline & Infarto agudo do miocárdio & 0 & 3 & 3 & 0,00 & 100,00 \\
\hline & QTc longo & 3 & 0 & 0 & 100,00 & 0,00 \\
\hline & QTc curto & 2 & 1 & 3 & 66,67 & 33,33 \\
\hline & Ritmo indeterminado & 1 & 0 & 1 & 100,00 & 0,00 \\
\hline & $\begin{array}{l}\text { Infarto do miocárdio recente, antigo ou de idade } \\
\text { indeterminada }\end{array}$ & 19 & 12 & 31 & 61,29 & 38,71 \\
\hline & Fibrilação atrial & 3 & 0 & 3 & 100,00 & 0,00 \\
\hline & Marca passo eletrônico (funcionamento normal) & 2 & 0 & 2 & 100,00 & 0,00 \\
\hline & Bloqueio de ramo esquerdo & 2 & 0 & 2 & 100,00 & 0,00 \\
\hline & Isquemia (possível) & 12 & 2 & 14 & 85,71 & 14,29 \\
\hline & Alteração de nível de ST (supra/infra) & 9 & 1 & 10 & 90,00 & 10,00 \\
\hline & Total & 53 & 19 & 72 & 73,16 & 23,69 \\
\hline \multirow{3}{*}{$\mathrm{V}$} & Impossível de analisar & & & & & \\
\hline & $\begin{array}{l}\text { Posicionamento incorreto de eletrodos, excesso de } \\
\text { artefatos }\end{array}$ & 9 & 0 & 9 & 100,00 & 0,00 \\
\hline & Total & 9 & 0 & 9 & 100,00 & 0,00 \\
\hline
\end{tabular}


Chama atenção, nos casos avaliados com o simulador de sinais eletrocardiográficos, a elevada sensibilidade (100\%) do eletrocardiógrafo interpretativo quando existe infra-desnível de segmento ST, compatível com quadros isquêmicos miocárdicos menos dramáticos que aqueles com supra-desnível, porém não necessariamente menos mórbidos. Além disso, considerando os casos simulados com o Metron, a sensibilidade $(93,8 \%)$ e a especificidade $(83,3 \%)$ para eletrocardiogramas compatíveis com isquemia miocárdica aguda são perfeitamente aceitáveis e, ainda, são consistentes com o desempenho do Cardiofax com pacientes, sumarizado nas Tabelas 4 e 5. Por outro lado, observou-se que as falhas diagnósticas em arritmias cardíacas são coerentes com aquelas já relatadas na literatura para os eletrocardiógrafos interpretativos (Kors e van Bemmel, 1990; Poon et al., 2005).

Conforme mencionado, uma das condições que sugere a existência de um processo isquêmico agudo do miocárdio é surgimento de um bloqueio de ramo esquerdo novo ou possivelmente novo (Anderson et al., 2007). O Metron ao ser programado

Tabela 5. Resultados dos diagnósticos eletrocardiográficos automáticos em pacientes, por categoria diagnóstica.

Table 5. Results of automated electrocardiograph diagnosis for patients, by diagnostic category.

\begin{tabular}{lccccr}
\hline \multirow{2}{*}{ Categoria } & \multicolumn{3}{c}{ Quantidades } & \multicolumn{3}{c}{$\begin{array}{c}\text { Porcentagem } \\
\text { (\%) }\end{array}$} \\
\cline { 2 - 6 } & Acertos & Erros & Total & Acertos & Erros \\
\hline Normal & 163 & 3 & 166 & 98,19 & 1,81 \\
Anormal & 124 & 23 & 147 & 84,35 & 15,65 \\
Limítrofe & 88 & 18 & 106 & 83,02 & 16,98 \\
Total & 375 & 44 & 419 & 89,50 & 10,50 \\
\hline
\end{tabular}

para produzir uma forma de onda compatível com esse distúrbio induziu o Cardiofax a informar o seguinte diagnóstico: "Ritmo sinusal, bloqueio inespecífico de condução intraventricular, desvio anormal do eixo para a esquerda, ECG anormal", enquanto que o eletrocardiografista "gold standard" entendeu tratar-se de "bloqueio divisional antero-superior esquerdo". Foi constatado que a forma de onda resultante do simulador (Figura 1) realmente não reproduz exatamente um bloqueio de ramo esquerdo (BRE), de modo que o autor concorda com o diagnóstico do "gold standard" e descarta a avaliação simulada desse parâmetro. É notável, ainda que a interpretação emitida pelo Cardiofax como "ritmo sinusal, bloqueio inespecífico de condução intraventricular, desvio anormal do eixo à esquerda, ECG anormal" possa ser considerada compatível com a interpretação de um BRE, embora utilize uma nomenclatura diversa daquela das diretrizes brasileiras.

A Figura 2 apresenta a situação em que o desnível de ST era tão pequeno que, a olho nu, o eletrocardiografista experiente considerou o exame como ritmo sinusal normal, embora o equipamento o tenha detectado e identificado corretamente como um distúrbio eletrocardiográfico.

Da exposição feita, fica evidente que o desempenho do eletrocardiógrafo interpretativo avaliado é adequado para detectar as alterações eletrocardiográficas clássicas associadas às síndromes isquêmicas agudas do miocárdio. Entretanto, existem outras anormalidades eletrocardiográficas, atípicas, mas que podem se relacionar com as altas indevidas (falhas diagnósticas) em síndromes coronarianas agudas (Pope et al., 2000). É possível avaliar a capacidade do eletrocardiógrafo interpretativo em

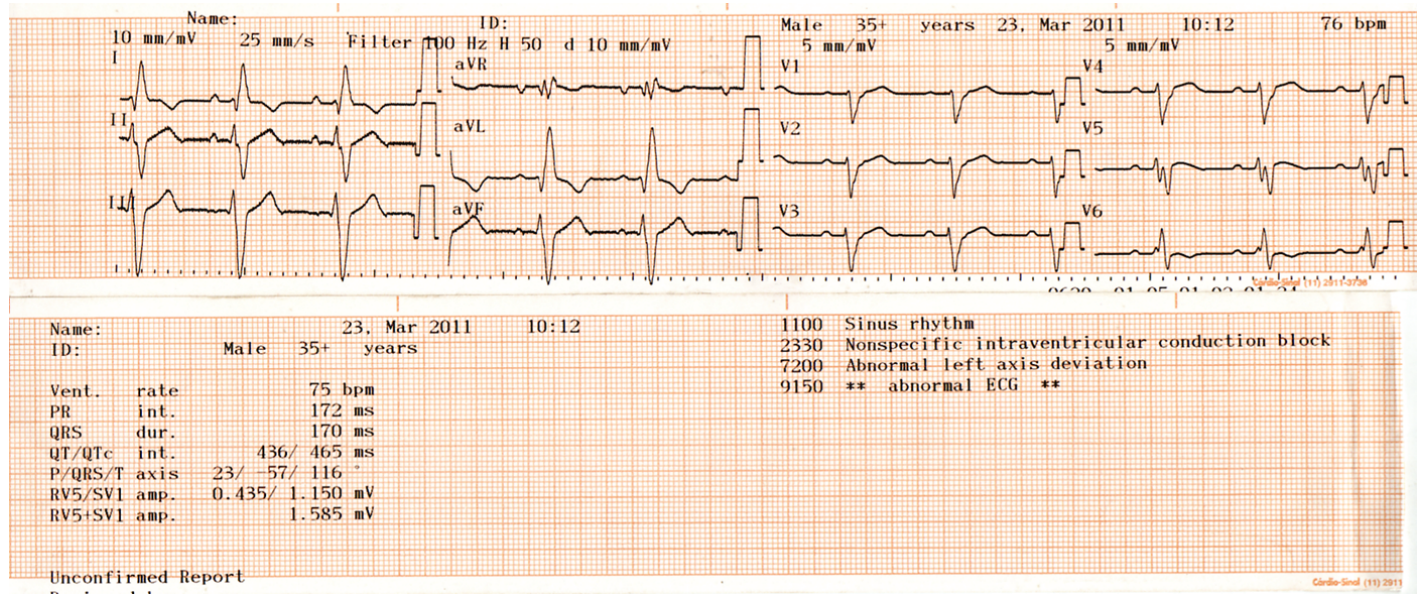

Figura 1. Eletrocardiograma interpretativo resultante dos sinais elétricos de um "bloqueio de ramo esquerdo" simulado.

Figure 1. Electrocardiogram interpretation of electrical signals resulting from a "left branch block" simulated. 
identificar essas situações (verdadeiros positivos), fazendo aproveitamento, especialmente, dos 419 ECGs obtidos de pacientes, apresentados na Tabela 4.

pelo equipamento como pequenos desníveis de segmento ST $(100 \%$ de capacidade de detecção Estas situações incluem os ECGs interpretados nos testes simulados), alterações inespecíficas de segmento ST ou anormalidades de onda T ( $84 \%$ de

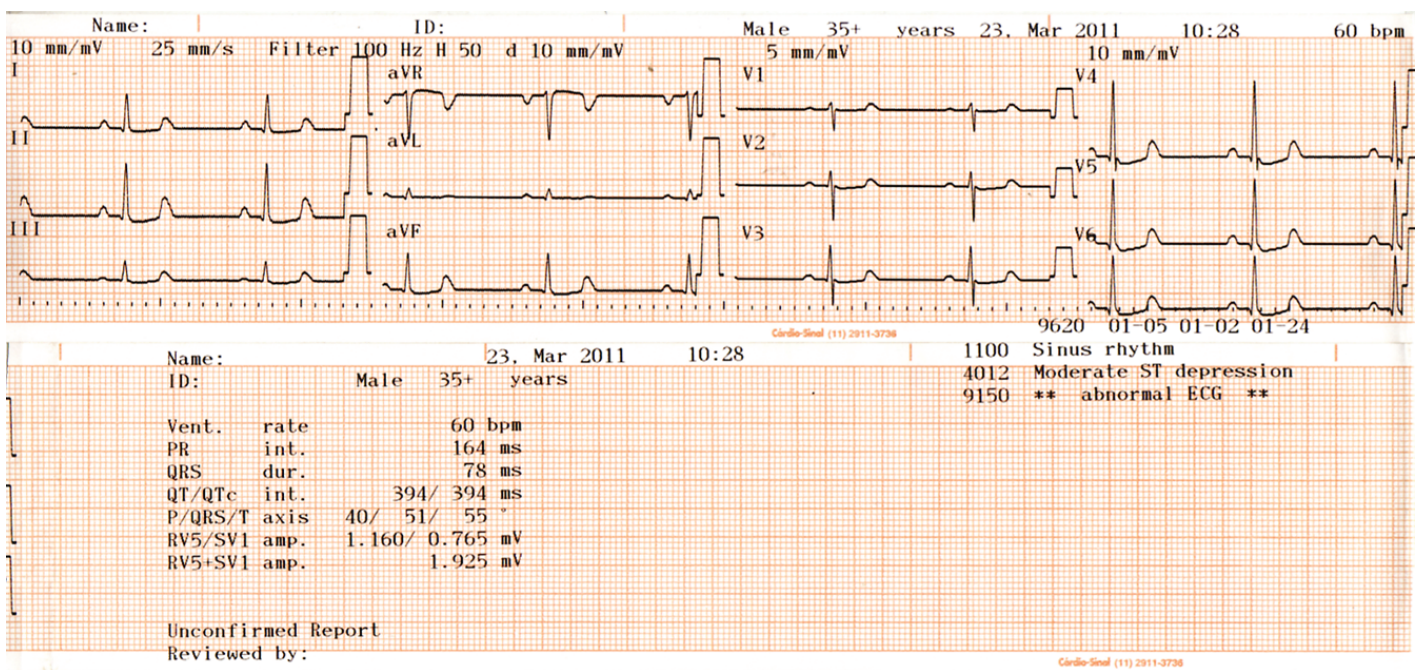

Figura 2. Eletrocardiograma interpretativo resultante dos sinais elétricos de um pequeno $(0,1 \mathrm{mV})$ infra-desnível de ST simulado, que induziu o eletrocardiografista "gold standard" a considerar o ECG como normal.

Figure 2. Electrocardiogram interpretation of electrical signals resulting from a small $(0.1 \mathrm{mV})$ below ST-elevation simulated, which induces the "gold standard" electrocardiographist to consider the ECG as normal.

Tabela 6. Resultados dos diagnósticos eletrocardiográficos de um serviço de telemedicina disponível em unidade médica semi-rural brasileira, por classe de significância.

Table 6. Results of the electrocardiographic diagnosis of a telemedicine service from a medical service of a semi-rural brazilian region, by class of significance.

\begin{tabular}{|c|c|c|c|c|c|c|}
\hline \multirow{2}{*}{ Classe } & \multirow{2}{*}{ Diagnóstico } & \multicolumn{3}{|c|}{ Quantidades } & \multicolumn{2}{|c|}{ Porcentagem (\%) } \\
\hline & & Acertos & Erros & Total & Acertos & Erros \\
\hline \multirow{3}{*}{ I } & Normal & & & & & \\
\hline & Ritmo sinusal & 4 & 2 & 6 & 66,67 & 33,33 \\
\hline & Total & 4 & 2 & 6 & 66,67 & 33,33 \\
\hline \multirow{7}{*}{ II } & Significância mínima & & & & & \\
\hline & Taquicardia sinusal & 3 & 0 & 3 & 100,00 & 0,00 \\
\hline & Arritmia sinusal & 0 & 1 & 1 & 0,00 & 100,00 \\
\hline & Disturbio de condução/ repolarização precoce & 2 & 4 & 6 & 33,33 & 66,67 \\
\hline & Anormalidade inespecífica de ST-T & 0 & 1 & 1 & 0,00 & 100,00 \\
\hline & Artefato & 0 & 1 & 1 & 0,00 & 100,00 \\
\hline & Total & 5 & 7 & 12 & 41,67 & 58,33 \\
\hline \multirow{5}{*}{ III } & Significância clínica indeterminada & & & & & \\
\hline & Hipertrofia/sobrecarga ventricular & 3 & 0 & 3 & 100,00 & 0,00 \\
\hline & Bloqueio completo de ramo direito & 1 & 1 & 2 & 50,00 & 50,00 \\
\hline & Extra-sístoles & 5 & 1 & 6 & 83,33 & 18,18 \\
\hline & Total & 9 & 2 & 11 & 81,82 & 18,18 \\
\hline \multirow{5}{*}{ IV } & Significância clínica definitiva & & & & & \\
\hline & Fibrilação atrial & 1 & 0 & 1 & 100,00 & 0,00 \\
\hline & Bloqueio de ramo esquerdo & 0 & 1 & 1 & 0,00 & 100,00 \\
\hline & Bloqueio AV avançado & 1 & 0 & 1 & 100,00 & 0,00 \\
\hline & Total & 2 & 1 & 3 & 66,67 & 333,33 \\
\hline \multirow{3}{*}{ V } & Impossível de analisar & & & & & \\
\hline & $\begin{array}{l}\text { Posicionamento incorreto de eletrodos, excesso de } \\
\text { artefatos }\end{array}$ & 0 & 0 & 0 & - & - \\
\hline & Total & 0 & 0 & 0 & - & - \\
\hline
\end{tabular}


capacidade de deteç̧ão nos testes com pacientes), anormalidades secundárias de segmento ST (hipertrofia ventricular esquerda [ $58 \%$ de capacidade de detecção nos testes com pacientes], bloqueio de ramo esquerdo $[100 \%$ de capacidade de detecção nos testes com pacientes] e variante de repolarização precoce $[82 \%$ de capacidade de detecção nos testes com pacientes]), cicatrizes miocárdicas prévias relatadas como infarto não agudo $(61 \%$ de capacidade de detecção nos testes com pacientes) e ECG normal (100\% de detecção nos testes com pacientes). É notável, ainda, a capacidade do equipamento em identificar nos pacientes as situações de ECGs verdadeiramente normais $(98,19 \%)$, anormais $(84,35 \%)$ e limítrofes $(83,02 \%)$, com uma taxa total de acertos de $89,50 \%$, conforme mostrada na Tabela 5. Além disso, no decorrer dos testes com pacientes não foram detectados casos de IAM, mas ocorreram três situações em que o eletrocardiógrafo interpretou como sendo infarto, correspondendo a falsos positivos, o que não prejudica a avaliação do equipamento, considerando que nas situações simuladas, compatíveis com IAM a acurácia foi aceitável.

Por outro lado, chama atenção o desempenho inadequado do serviço de telemedicina disponível no hospital semi-rural (Tabelas 6 e 7) utilizado para calcular o tamanho da amostragem para o teste do Cardiofax. Isso pode ser demonstrado pelo diagnóstico incorreto de um traçado eletrocardiográfico como sendo um "bloqueio de ramo esquerdo de $2^{\circ}$ grau" (Figura 3). Segundo o eletrocardiografista "gold standard" que avaliou os ECGs neste trabalho, esse registro corresponde a uma "sobrecarga de ventrículo esquerdo associado a uma área inativa septal". Tal erro não foi observado com as interpretações feitas pelo programa ECAPS 12C do Cardiofax, conforme se constata comparando-se as Tabelas 4 e 6 , onde o equipamento acertou todos os 3 casos de "bloqueio de ramo esquerdo" que se lhe apresentaram (dentre as 419 amostras obtidas em pacientes), enquanto que o serviço de telemedicina observado errou (falso positivo) a única interpretação de bloqueio de ramo esquerdo (dentre 26 amostras de pacientes). Como um bloqueio de ramo esquerdo (BRE) novo ou presumivelmente novo é uma das condições a ser levada em conta no diagnóstico de um infarto agudo do miocárdio, essa situação é preocupante. Além disso, nota-se, no citado serviço de telemedicina, que, nesse caso, o exame chegou às 09:45 h e teve seu resultado enviado às 10:06 $\mathrm{h}$, ou seja, um tempo resposta de 21 minutos para disponibilizar a interpretação, que é significativamente maior que aquele do Cardiofax (tipicamente alguns segundos). Como a maioria dos óbitos por infarto ocorre na primeira hora do início dos sintomas (Oliveira e Spiandorello, 2001), esses
Tabela 7. Resultados dos diagnósticos de um serviço de telemedicina disponível em unidade médica semi-rural brasileira, por categoria diagnóstica.

Table 7. Results of the electrocardiographic diagnosis of a telemedicine service from a medical service of a semi-rural brazilian region, by diagnostic category.

\begin{tabular}{lccccc}
\hline \multirow{2}{*}{ Categoria } & \multicolumn{2}{c}{ Quantidades } & \multicolumn{3}{c}{$\begin{array}{c}\text { Porcentagem } \\
(\%)\end{array}$} \\
\cline { 2 - 6 } & Acertos & Erros & Total & Acertos & Erros \\
\hline Normal & 6 & 1 & 7 & 85,17 & 14,29 \\
Anormal & 6 & 1 & 7 & 85,17 & 14,29 \\
Limítrofe & 2 & 10 & 12 & 16,67 & 83,33 \\
Total & 14 & 12 & 26 & 53,85 & 46,15 \\
\hline
\end{tabular}

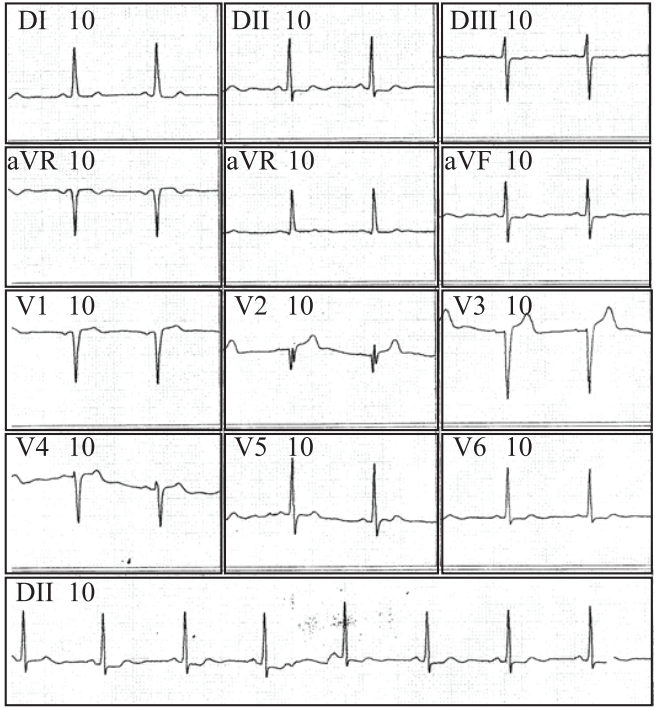

LAUDO DO SISTEMA DE TELEMEDICINA

Ritmo: Sinusal

Frequência cardíaca: $65 \mathrm{bpm}$

SÂQRS: $35^{\circ}$

Conclusão: Bloqueio do ramo esquerdo do $2^{\circ}$ grau

Figura 3. Bloqueio de ramo esquerdo do $2^{\circ}$ grau erroneamente diagnosticado por sistema de telemedicina disponível para uma unidade médica semi-rural brasileira (por questões éticas, as informações que permitissem identificar a empresa, o hospital, o paciente, interpretador e a tecnologia foram suprimidas após a digitalização do documento). Figure 3. Left bundle branch block of 2nd degree misdiagnosed by a system of telemedicine from a medical service of a semi-rural brazilian region, (for ethical reasons, information that might have identified the company, the hospital, the patient, the interpreter and the technology was suppressed after scanning the document).

21 minutos perdidos, aguardando o diagnóstico, podem fazer a diferença entre a vida e a morte do paciente, caso, realmente, o diagnóstico da telemedicina estivesse correto. Mais ainda, considerando que esse falso bloqueio de ramo esquerdo poderia reforçar uma hipótese diagnóstica de isquemia miocárdica aguda, se o clínico geral tiver aceitado o diagnóstico 
da telemedicina, a sua conduta médica talvez não tenha sido a mais apropriada para a situação clínica presente no paciente.

\section{Conclusões}

Em função dos resultados dos testes realizados neste trabalho, é possível afirmar que é adequado o uso do eletrocardiógrafo interpretativo Cardiofax 9620 da Nihon Kohden como elemento de um sistema computacional de auxílio ao diagnóstico em síndrome coronariana aguda. Isto é coerente com as diretrizes da American Heart Association (AHA) e do American College of Cardiology $(A C C)$ quando recomendam que sejam feitos ECGs no atendimento pré-hospitalar, na triagem dos pacientes com dor torácica sugestiva de síndrome coronariana aguda, considerando razoável o uso de eletrocardiógrafos que disponham de algoritmos validados de interpretação gerados por computador para estes casos, com o nível de evidência B, classe IIa (Anderson et al., 2007). Este nível de evidência corresponde a avaliações feitas em estratos populacionais limitados, enquanto que a classe especificada indica que os benefícios da adoção do procedimento superam em muito os riscos. Essas associações norte-americanas de Cardiologia, sugerem as seguintes frases para o uso de procedimentos médicos com o nível de evidência B, classe IIa: "é razoável", "pode ser útil/efetivo/benéfico", "é provavelmente recomendado ou indicado" (Anderson et al., 2007).

Em que pese o desempenho aceitável do eletrocardiógrafo interpretativo testado em situações compatíveis com síndromes coronarianas agudas, o uso indiscriminado desse tipo de equipamento é ainda objeto de controvérsia na classe médica brasileira, possivelmente pelo seu desempenho sofrível em arritmias cardíacas relatado na literatura (Kors e van Bemmel, 1990; Poon et al., 2005). Entretanto, é necessário considerar que, tipicamente, um paciente com síndrome coronariana aguda é atendido inicialmente em hospitais gerais por um clínico com limitados conhecimentos de eletrocardiografia e não por um cardiologista. Nessas condições, onde são importantes achados tais como desvios de segmento ST, inversão de onda $\mathrm{T}$ e a presença de onda $\mathrm{Q}$, existe recomendação na literatura estrangeira no sentido de que o clínico geral reavalie sua interpretação do ECG quando o eletrocardiógrafo interpretativo reportar essas condições, como uma forma de reduzir a taxa de falso-negativos (Jensen et al., 2005). Isso, juntamente com as mencionadas recomendações da $A H A / A C C$ quanto ao benefício do uso de eletrocardiógrafos interpretativos nesses casos (Anderson et al., 2007), torna interessante o emprego desse tipo de equipamento no manejo inicial das doenças isquêmicas agudas do coração, o que está perfeitamente dentro do escopo deste trabalho.

Adicionalmente, este trabalho identificou uma deficiência qualitativa - que pode ser isolada ou mesmo temporária - em um serviço de telemedicina privado disponível em uma unidade médica semi-rural do Brasil. Isto sugere que tal tipo de serviço, especialmente quando dedicado à interpretação remota de eletrocardiogramas, deva ser submetido a processos de certificação e avaliação periódicos por uma entidade pública competente, e que, para tanto, sejam estabelecidos parâmetros de desempenho a serem obedecidos, de modo a viabilizar uma sistemática de fiscalização da telemedicina eletrocardiográfica, para que este método possa, efetivamente, contribuir para a redução da mortalidade por doença cardiovascular.

\section{Referências}

Anderson JL, Adams CD, Antman EM, Bridges CR, Califf RM, Casey DE, Chavey II WE, Fesmire FM, Hochman JS, Levin TN, Lincoff AM, Peterson ED, Theroux P, Wenger NK, Wright RS. ACC/AHA 2007 Guidelines for the management of patients with unstable angina/non-ST-elevation myocardial infarction: executive summary. Circulation. 2007; 116:803-77. http://dx.doi.org/10.1161/CIRCULATIONAHA.107.185752

Braile DM, Godoy MF. Cálculos estatísticos básicos para testes diagnósticos [Internet]. Brasil; 1999 [acesso em 2011 jan. 20]. Disponível em: www.braile.com.br/download/ download.htm

Deccache W. Eletrocardiograma revisitado e facilitado. Rio de Janeiro: Revinter; 2006.

Farrell RM, XueJQ,Young BJ. Enhanced rhythm analysis for resting ECG using spectral and time-domain techniques. Computers in Cardiology. 2003; 30:733-6.

Frieden J, Ira LR. ECG case studies: 100 new challenging ECGs. 2nd ed. New York: Medical Examination; 1974.

Hampton JR, Adlam D. ECG na prática. 5. ed. Rio de Janeiro: Elsevier; 2009.

Instituto Nacional de Cardiologia. INC: Relatório de gestão do exercício 2008 [Internet]. Brasil; 2008 [acesso em 2011 mar. 06]. Disponível em: http://www.inc.saude.gov.br/ paginas/transparencia_publica/Processos_Contas/relat2008/ Relatorio_Gest\%C3\%A3o_2008.pdf.

Instituto Dante Pazzanese de Cardiologia. Laboratório de epidemiologia e estatística [Internet]. São Paulo, Brasil: LEE; 2010. [acesso em 2010 nov. 10]. Disponível em: www.lee.dante.br/pesquisa.html

Jakobsson A, Pahim O, Reichert L, Edenbrandt L, Nilsson B. On the consistency of ECG reports from two different computer-based ECG records. Clinical Physiology. 1989; 9:289-94. PMid:2663333. http://dx.doi. org/10.1111/j.1475-097X.1989.tb00981.x 
Jensen MSA, Thomsen JL, Jensen SE, Laurentzen T, Engber M. Electrocardiogram interpretation in general practice. Family Practice. 2005; 22(1):100-13.

Kligfield P. Automated analysis of ECG rhytm. Clinical Window Web Journal: Window on Cardiology. 2005; 5:20.

Kors JA, Van Bemmel JH. Classification methods for computerized interpretation of the electrocardiogram. Methods of Information in Medicine.1990; 29:336.

Nihon Kohden. Users's guide: ECG interpretation program ECAPS 12C. 9th ed. Tokyo: Nihon Kohden; 2009.

Oliveira EM, Spiandorello FSA. Síndromes coronarianas agudas: protocolo de avaliação e manejo inicial de pacientes com dor torácica. Revista Científica da AMECS. 2001; 1-2.

Poon K, Okin PM, KligfieldP. Diagnostic performance of a computer-based ECG rhythm algorithm. Journal of Electrocardiology. 2005; 38:235-8. PMid:16003708. http:// dx.doi.org/10.1016/j.jelectrocard.2005.01.008

Pope HJ, Aufderheide TP, Ruthazer R, Woolard RH, Feldman JA, Beshansky JR, Griffith JL, Selker HP. Missed diagnoses of acute cardiac ischemia in the emergency department. New England Journal of Medicine. 2000; 342(16):1163-70. PMid:10770981. http:// dx.doi.org/10.1056/NEJM200004203421603

Rautaharju PM. Will the electrocardiograph replace the electrocardiographers. Journal of Electrocardiology. 1993; 26:158-63.

Reddy BR, Taha B, Swiryn S, Silbermann R, Childers R. Prospective evaluation of a microprocessor assisted cardiac rhythm algorithm: results from one clinical center. Journal of Electrocardiology. 1998; 30:28-33. http://dx.doi. org/10.1016/S0022-0736(98)80016-2

Selker HPI, McCarthy BD, Beshansky R, D’Agostino RB. Missed diagnoses of acute myocardial infarction in the emergency department: Results from a multicenter study. Annals of Emergency Medicine. 1993; 22(3):579-82. http:// dx.doi.org/10.1016/S0196-0644(05)81945-6
Snyder CS, Fenrich AL, Friedman RA, Macias C, O’Reilly $\mathrm{K}$, Kertsez NJ. The emergency department versus the computer: which is the better electrocardiographer? Pediatric Cardiology. 2003; 24:364-8. PMid:12457259. http://dx.doi. org/10.1007/s00246-002-0332-Z

Sociedade Brasileira de Cardiologia. Diretrizes da Sociedade Brasileira de Cardiologia sobre análise e emissão de laudos eletrocardiográficos. Arquivos Brasileiros de Cardiologia. 2009; 93(supl. 2):1-19.

Spodick DH, Bishop RL. Computer treason: intraobserver variability of an electrocardiograph computer system. American Journal of Cardiology. 1997;80:102-3. http:// dx.doi.org/10.1016/S0002-9149(97)00296-8

Taha B, Reddy S, Xue Q, Swiryn S. Automated discrimination between atrial fibrillation and atrial flutter in the resting 12-lead electrocardiogram. Journal of Electrocardiology. 2000; 33:123-5. PMid:11265711. http:// dx.doi.org/10.1054/jelc.2000.20303

Thomson A, Mitchell S, Harris PJ. Computerized electrocardiographic interpretation: an analysis of clinical utility in 5110 electrocardiograms. Medical Journal of Australia. 1989; 151:428-30. PMid:2593957.

Willems JL, Abreu-Lima C, Arnaud P, van Bemmel JH, Brohet C, Degani R, Denis B, Gehring J, Graham I, van Herpen G, Machado H, Macfarlane PW, Michaelis J, Moulopoulos SD, Rubel P, Zywietz C. The diagnostic performance of computer programs for the interpretation of electrocardiograms. New England Journal of Medicine. 1991; 325:1767-73. PMid:1834940. http://dx.doi.org/10.1056/ NEJM199112193252503

Wooley D, Henck M, Luck J. Comparison of electrocardiogram interpretations by family physicians, a computer and a cardiology service. Journal of Family Practice. 1992; 34:428-32. PMid:1556536.

\footnotetext{
Autores

Manoel Gadêlha de Freitas Junior*, Denys Emilio Campion Nicolosi, Francisco Faustino de Albuquerque Carneiro de França

Programa de Pós-Graduação em Medicina/Tecnologia e Intervenção em Cardiologia, Instituto Dante Pazzanese de Cardiologia - IDPC, Universidade de São Paulo - USP, Av. Dr. Dante Pazzanese, 500, Vila Mariana, CEP 04012-909, São Paulo, SP, Brasil.
} 